Document downloaded from:

http://hdl.handle.net/10251/139654

This paper must be cited as:

Burgos-Simon, C.; Cortés, J.; Debbouche, A.; Villafuerte, L.; Villanueva Micó, RJ. (2019). Random fractional generalized Airy differential equations: A probabilistic analysis using mean square calculus. Applied Mathematics and Computation. 352:15-29.

https://doi.org/10.1016/j.amc.2019.01.039

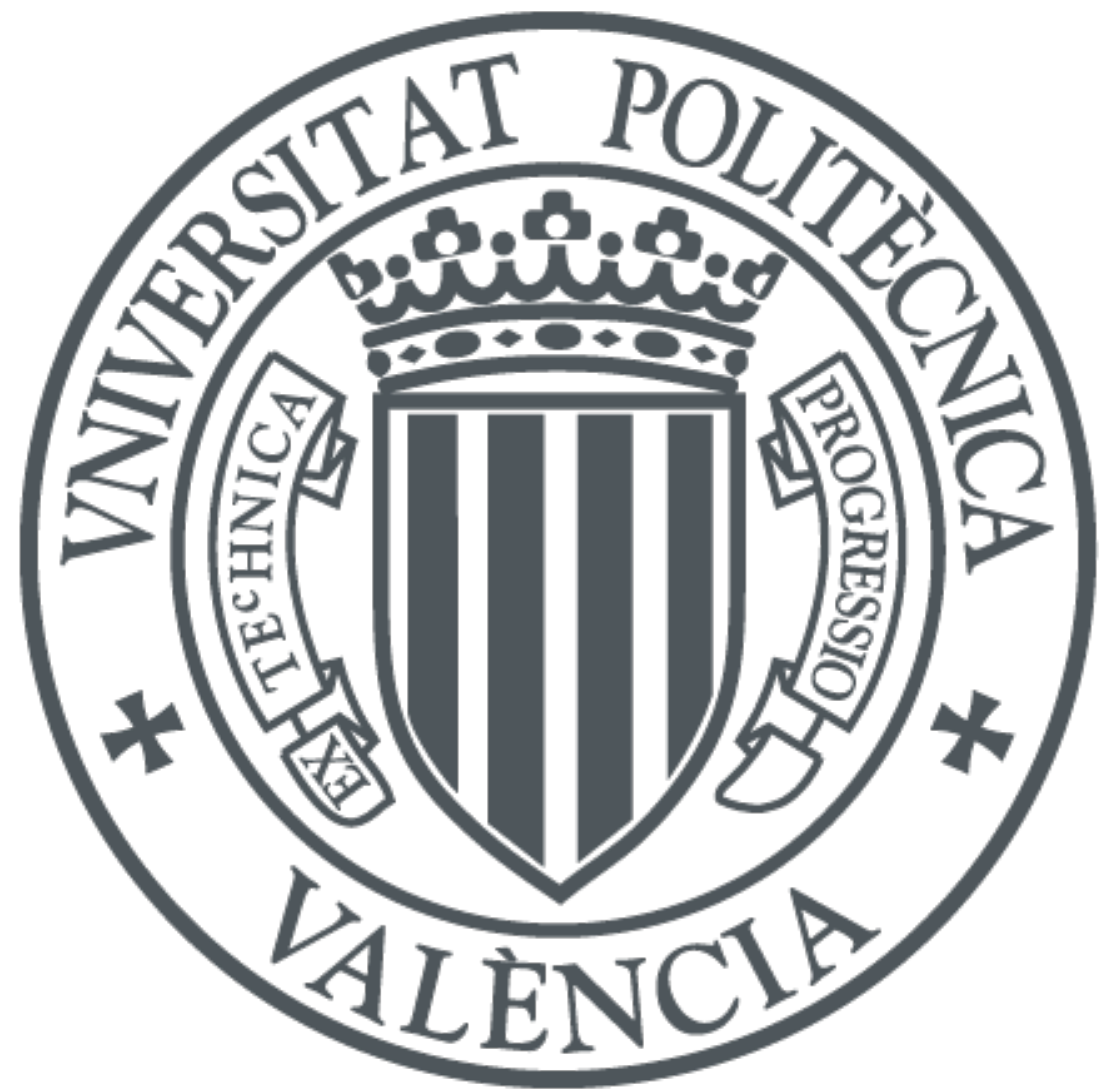

The final publication is available at

https://doi.org/10.1016/10.1016/j.amc.2019.01.039

Copyright Elsevier

Additional Information 


\title{
Random fractional generalized Airy differential equations: A probabilistic analysis using mean square calculus
}

\author{
C. Burgos ${ }^{\mathrm{a}}$, J.-C. Cortés ${ }^{\mathrm{a}}$, A. Debbouche ${ }^{\mathrm{b}, *}$, L. Villafuerte $^{\mathrm{c}}$, R.-J. Villanueva ${ }^{\mathrm{a}}$ \\ ${ }^{a}$ Instituto Universitario de Matemática Multidisciplinar, \\ Universitat Politècnica de València, \\ Camino de Vera s/n, 46022, Valencia, Spain \\ ${ }^{b}$ Department of Mathematics, Guelma University, Guelma 24000, Algeria \\ ${ }^{c}$ Department of Mathematics, \\ University of Texas at Austin, USA
}

\begin{abstract}
The aim of this paper is to study a generalization of fractional Airy differential equations whose input data (coefficient and initial conditions) are random variables. Under appropriate hypotheses assumed upon the input data, we construct a random generalized power series solution of the problem and then we prove its convergence in the mean square stochastic sense. Afterwards, we provide reliable explicit approximations for the main statistical information of the solution process (mean, variance and covariance). Further, we show a set of numerical examples where our obtained theory is illustrated. More precisely, we show that our results for the random fractional Airy equation are in full agreement with the corresponding to classical random Airy differential equation available in the extant literature. Finally, we illustrate how to construct reliable approximations of the probability density function of the solution stochastic process to the random fractional Airy differential equation by combining the knowledge of the mean and the variance and the Principle of Maximum Entropy.
\end{abstract}

Keywords: Caputo fractional derivative, Random analysis, Airy differential equations, Mean square calculus, Stochastic simulations, Principle of Maximum Entropy.

\section{Introduction}

Differential equations (DEs) have demonstrated to be useful mathematical tools to model complex problems in different scientific realms such as Physics, Chemistry, Economics, Epidemiology, etc. Here we want to underscore two different variations of the classical formulation of DEs that have been proposed to get a better understanding of real phenomena via their mathematical modelling. The first approach is based upon fractional differential equations (FDEs) while the second is formulated by random differential equations (RDEs).

\footnotetext{
*Corresponding author

Email addresses: clabursi@posgrado.upv.es (C. Burgos), jccortes@imm.upv.es (J.-C. Cortés), amar_debbouche@yahoo.fr (A. Debbouche), altuzar@math.utexas.edu (L. Villafuerte), rjvillan@imm.upv.es (R.-J. Villanueva)
} 
On the one hand, FDEs are generalizations of DEs to an arbitrary order of the involved derivatives. These kind of equations have been applied to describe the dynamics of complex phenomena related, for example, to visco-elastic materials in Engineering, subdiffusive processes in Physics, epidemics in Medicine, etc. The success of FDEs to deal with this type of problems lies in their ability to account for memory effects $[1,2,3]$. Some recent papers dealing with analytic and numerical techniques to study interesting problems about FDEs can be found in $[4,5,6,7,8,9]$, for example.

On the other hand, RDEs are DEs whose formulation involves uncertain information in their data. Examples of RDEs are those whose coefficients, unhomogeneous term and/or initial/boundary conditions are random variables and/or stochastic processes. The formulation of RDEs appears in a natural manner when considering uncertainty stemming from the measurements required to set the values of their data and the complexity of the phenomena under study as well. It is important to point out that there exist different approaches to deal with the rigorous study of RDEs. These approaches are heavily related to the type of stochastic convergence used to define the derivative involved in the formulation of the corresponding RDE, namely, mean square and almost surely convergences (which are strong-type stochastic convergences) and probability and distribution convergences (which are weak-type stochastic convergences). In general, it is better to establish results for RDEs using mean square or almost surely convergences rather than using weaker stochastic convergences since strong convergence entails weak convergence. Some interesting contributions dealing with theory and applications to RDEs can be found in $[10,11,12,13,14]$, for instance. Throughout our subsequent study, we will use mean square stochastic convergence only because of its key properties that will be apparent later. Accordingly, it is also worth pointing out that relevant results belonging to the deterministic fractional calculus have been recently extended to the stochastic setting taking advantage of the mean square stochastic convergence and its key properties [15]. These results include the definition of the random mean square Riemann-Liouville integral and of the random mean square Caputo derivative as well as their characterizations in terms of the associated correlation function.

In this paper, we combine FDEs and RDEs to study an important class of random fractional differential equations (RFDEs) that generalizes the classical Airy-type differential equations both in the random and in the fractional senses. This fractional differential equation has been studied in the deterministic setting in reference [2, p.232] and we will extend the analysis to the random framework. Specifically, we will deal with the following random fractional generalized Airy-type initial value problem (IVP)

$$
\left\{\begin{array}{cl}
\left({ }^{C} D_{0^{+}}^{\alpha} Y\right)(t)-B t^{\beta} Y(t) & =0, \quad t>0, \quad n-1<\alpha \leq n, \quad \beta>0, \\
Y^{(j)}(0) & =A_{j}, \quad j=0,1, \ldots, n-1,
\end{array}\right.
$$

where

$$
\left({ }^{C} D_{0^{+}}^{\alpha} Y\right)(t):=\frac{1}{\Gamma(n-\alpha)} \int_{0}^{t}(t-u)^{n-\alpha-1} Y^{(n)}(u) \mathrm{d} u
$$

is the mean square random Caputo fractional derivative of order $\alpha$ of the stochastic process $Y(t)$ (see [15] and references therein). Notice that if $\alpha=2$ and $\beta=1$, then (1) becomes the classical Airy differential equation with initial conditions $A_{0}$ and $A_{1}$. In our general setting to (2), $n \geq 1$ is a fixed integer such that $n=-\lfloor-\alpha\rfloor$ being $\lfloor\cdot\rfloor$ the floor function. The initial conditions $A_{j}$, $j=0,1, \ldots, n-1$, and coefficient $B$ are assumed to be independent real random variables defined in the Hilbert space $\left(\mathrm{L}_{2}(\Omega),\langle\cdot, \cdot\rangle\right)$. For the sake of clarity, we recall that the elements of $\mathrm{L}^{2}(\Omega)$ 
are $X: \Omega \longrightarrow \mathbb{R}$ such that $\mathbb{E}\left[X^{2}\right]<+\infty$, being $\mathbb{E}[\cdot]$ the expectation operator, and the inner product is defined by $\langle X, Y\rangle=\mathbb{E}[X Y], X, Y \in \mathrm{L}^{2}(\Omega)$. Therefore, $\|X\|_{2}=+\sqrt{\langle X, X\rangle}=\left(\mathbb{E}\left[X^{2}\right]\right)^{1 / 2}$. This norm is usually termed the mean square norm and from it one infers the so-called mean

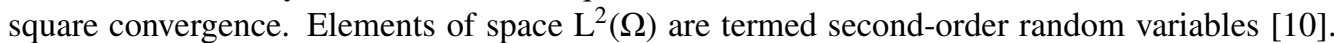
As usual, the triplet $(\Omega, \mathcal{F}, \mathbb{P})$ denotes a common underlying complete probability space for the random variables $A_{j}, j=0,1, \ldots, n-1$ and $B$. Notice that every random variable with finite variance belongs to $\mathrm{L}^{2}(\Omega)$. This class of random variables is met in the most of physical problems involving randomness where usually the variability (variance) is finite. We also recall that given $\mathcal{T} \subset \mathbb{R}$, then $Z(t)$ is called a second-order stochastic process if $Z(t) \equiv\{Z(t): t \in \mathcal{T}\}$ is a secondorder random variable for every $t \in \mathcal{T}$. Naturally, $Z(t)$ is said to be mean square differentiable at $t_{0} \in \mathcal{T} \subset \mathbb{R}$ if $\lim _{h \rightarrow 0}\left\|\frac{Z\left(t_{0}+h\right)-Z\left(t_{0}\right)}{h}-Z^{\prime}\left(t_{0}\right)\right\|_{2}=0$, and in that case, the second-order random variable $Z^{\prime}\left(t_{0}\right)$ is called its mean square first derivative at $t=t_{0}$. Higher-order mean square derivatives are defined in an analogous manner. Throughout this paper, the derivatives will be denoted as $Z^{(m)}(t)$ or $\frac{\mathrm{d}^{m} Z(t)}{\mathrm{d} t^{m}}$. In [10] one presents a quite extensive overview of the mean square random calculus including its main operational rules to rigorously handle RDEs.

The mathematical study of some classes of RFDEs using mean square stochastic calculus has been presented in some recent contributions $[15,16,17,18,19,20]$. It must be also highlighted other recent interesting contributions to deal with RFDEs using different approaches like differential inclusions [21] and generalized polynomial chaos [22].

The Airy differential equations and its related functions [23] were shown best applicability in several fields such as fluid mechanics, elasticity, quantum physics and so on. It has been studied separately in the context of deterministic FDEs [24, 25, 26] and in the RDEs [27], however to the best of our knowledge, its mathematical study combining both fractional and random calculus, i.e., treating it as a RFDEs has not been undertaken yet, which is the motivation of this scientific contribution.

The main goal of this article is to establish conditions on random variables $A_{j}, j=0,1, \ldots, n-$ 1 , and $B$ in order to construct a mean square solution to problem (1) in the following form

$$
Y(t)=\sum_{j=0}^{n-1} Y_{j}(t) \quad \text { with } \quad Y_{j}(t)=\sum_{m=0}^{\infty} X_{m, j} t^{\gamma m+j} .
$$

In the above expression, $X_{m, j}$ is a sequence of second-order random variables to be determined, and $\gamma:=\alpha+\beta$. Observe that $\gamma>n-1$ because $n-1<\alpha \leq n$ and $\beta>0$. As the solution of random IVP (1) is a stochastic process, an important target is to compute its statistical properties as well. Specifically, as our study is based on mean square random calculus corresponding to $\mathrm{L}^{2}(\Omega)$, we will construct approximations for the two first statistical moments (the mean and the variance) of the solution stochastic process $Y(t)$. At this point it is worth pointing out that in this way we will give our results in the biggest $\mathrm{L}^{p}(\Omega)$-type Lebesgue space, since it is known that $\mathrm{L}^{q}(\Omega) \subset \mathrm{L}^{p}(\Omega), 1 \leq p \leq q \leq \infty$, and taking $p=2$ is guaranteed the existence of variance (or equivalently of randomness). As an interesting application of obtaining approximations of the mean and of the variance, we take advantage of Principle of Maximum Entropy to construct the probability density function of the solution.

This paper is organized as follows: In Section 2, first we construct a random generalized power series solution of the form (3) to the random fractional IVP (1) and then we provide sufficient conditions on the coefficient $B$ and the initial conditions $A_{j}, 0 \leq j \leq n-1$, in order to guarantee the mean square convergence of that random series. Section 3 is addressed to compute the main statistical properties of the solution stochastic process, namely, the mean, the 
variance and the covariance. Section 4 is devoted to show a set of numerical examples where our theoretical results are illustrated. Section 5 shows, by means of a detailed example, how to construct reliable approximations of the probability density function of the solution stochastic process to the random fractional Airy differential equation by combining the knowledge of the mean and of the variance together with the Principle of Maximum Entropy. In this manner we are able to provide a full probabilistic description of the solution stochastic process at each time instant. Our conclusions are shown in Section 6.

\section{Constructing the solution stochastic process}

As it will be seen right now, we will obtain the solution to the random fractional IVP (1) following a constructive reasoning. This process will require to legitimate certain operational rules, in the mean square sense. For the sake of clarity, we start this section by rigorously proving these rules. To this purpose, we will apply [10, property (4.126), p. 96] to calculate the mean square derivative of the product of a differentiable deterministic function and a mean square differentiable stochastic process, and [28, Th. 3.1, p. 1260] to differentiate a series of mean square stochastic processes.

Our first step will be to rigorously legitimate that the first mean square derivative of the stochastic process $Y_{j}(t)$, defined in (3) for $t>0$, is given by

$$
\frac{\mathrm{d}}{\mathrm{d} t}\left[Y_{j}(t)\right]=\sum_{m=0}^{\infty} X_{m, j} \frac{\mathrm{d}}{\mathrm{d} t}\left[t^{\gamma m+j}\right], \quad j=0,1, \ldots, n-1 .
$$

Indeed, take $t_{0}>0$ fixed and for each $j$, let $U_{m}(t)=X_{m, j} t^{\gamma m+j}$ being $X_{m, j} \in \mathrm{L}^{2}(\Omega)$. Then applying [10, property (4.126), p. 96] to $f(t)=t^{\gamma m+j}$ and $X(t)=X_{m, j}$, one gets that $U_{m}(t)$ is mean square differentiable at $t_{0}$ being $\left.\frac{\mathrm{d}}{\mathrm{d} t}\left[U_{m}(t)\right]\right|_{t=t_{0}}=\left.X_{m, j} \frac{\mathrm{d}}{\mathrm{d} t}\left[t^{m \gamma+j}\right]\right|_{t=t_{0}}$ its mean square derivative for every $m \geq 0$. Moreover, if $X_{m, j} \in \mathrm{L}^{2}(\Omega)$ then clearly the sequence of stochastic processes $\left\{U_{m}(t)\right.$ : $m \geq 0\}$ is mean square continuous at $t_{0}$. Take $\mathcal{T}$ an interval in $\mathbb{R}^{+}$and define $U(t)=\sum_{m \geq 0} U_{m}(t)$ If this latter sum is mean square convergent and $\sum_{n \geq 0} U_{m}^{\prime}(t)$ converges uniformly in the mean square sense for each $t \in \mathcal{T}$, then by [28, Th. 3.1, p. 1260] it is guaranteed that (4) holds. At this point is important to underline that we have implicitly assumed that $X_{m, j} \in \mathrm{L}^{2}(\Omega)$. Later we will compute explicitly coefficients $X_{m, j}$ in terms of $A_{j}, j=0,1, \ldots, n-1$ and $B$, and then assuming appropriate hypotheses on input data we will check that $X_{m, j} \in \mathrm{L}^{2}(\Omega)$. Following an analogous reasoning, it can easily be shown that

$$
\frac{\mathrm{d}^{n}}{\mathrm{~d} t^{n}}\left[Y_{j}(t)\right]=\sum_{m=0}^{\infty} X_{m, j} \frac{\mathrm{d}^{n}}{\mathrm{~d} t^{n}}\left[t^{\gamma m+j}\right], \quad n \geq 1,
$$

being $n$ a fixed positive integer.

Using the fractional derivative of the power function $t^{\nu}$ given by [29, Example 3.1]

$$
{ }^{C} D_{0^{+}}^{\alpha} t^{v}=\left\{\begin{array}{ccc}
t^{\nu-\alpha} \frac{\Gamma(v+1)}{\Gamma(v+1-\alpha)} & \text { if } \quad v>n-1, \\
0 & \text { if } \quad v=0,1, \ldots, n-1,
\end{array} \quad n=-\lfloor-\alpha\rfloor, \alpha>0,\right.
$$


and (5), one derives

$$
\begin{aligned}
\left({ }^{C} D_{0^{+}}^{\alpha} Y\right)(t) & =\left({ }^{C} D_{0^{+}}^{\alpha} \sum_{j=0}^{n-1} Y_{j}\right)(t) \\
& =\sum_{j=0}^{n-1}\left({ }^{C} D_{0^{+}}^{\alpha} Y_{j}\right)(t) \\
& =\sum_{j=0}^{n-1}\left(\frac{1}{\Gamma(n-\alpha)} \int_{0}^{t}(t-u)^{n-\alpha-1} \frac{\mathrm{d}^{n}}{\mathrm{~d} u^{n}}\left[Y_{j}(u)\right] \mathrm{d} u\right) \\
\text { by (5) } \quad & \sum_{j=0}^{n-1}\left(\frac{1}{\Gamma(n-\alpha)} \int_{0}^{t}(t-u)^{n-\alpha-1} \sum_{m=0}^{\infty} X_{m, j} \frac{\mathrm{d}^{n}}{\mathrm{~d} u^{n}}\left[u^{\gamma m+j}\right] \mathrm{d} u\right) \\
& =\sum_{j=0}^{n-1}\left(\sum_{m=0}^{\infty} X_{m, j} \frac{1}{\Gamma(n-\alpha)} \int_{0}^{t}(t-u)^{n-\alpha-1} \frac{\mathrm{d}^{n}}{\mathrm{~d} u^{n}}\left[u^{\gamma m+j}\right] \mathrm{d} u\right) \\
& =\sum_{j=0}^{n-1}\left(\sum_{m=0}^{\infty} X_{m, j}{ }^{C} D_{0^{+}}^{\alpha} \gamma^{\gamma m+j}\right) \\
\text { by (6) } & =\sum_{j=0}^{n-1} \sum_{m=1}^{\infty} X_{m, j} \frac{\Gamma(\gamma m+j+1)}{\Gamma(\gamma m+j+1-\alpha)} t^{\gamma m+j-\alpha} \\
& =\sum_{m=0}^{\infty} \sum_{j=0}^{n-1} X_{m+1, j} \frac{\Gamma(\gamma(m+1)+j+1)}{\Gamma(\gamma(m+1)+j+1-\alpha)} t^{\gamma(m+1)+j-\alpha} .
\end{aligned}
$$

Notice that when applying (5), we have used that $\gamma=\alpha+\beta>n-1$.

Hence, taking into account that $\gamma=\alpha+\beta$, one obtains

$$
\begin{aligned}
\left({ }^{C} D_{0^{+}}^{\alpha} Y\right)(t)-B t^{\beta} Y(t) & =\sum_{m=0}^{\infty} \sum_{j=0}^{n-1} X_{m+1, j} \frac{\Gamma(\gamma(m+1)+j+1)}{\Gamma(\gamma(m+1)+j+1-\alpha)} t^{\gamma(m+1)+j-\alpha} \\
& -B t^{\beta} \sum_{m=0}^{\infty} \sum_{j=0}^{n-1} X_{m, j} t^{\gamma m+j} \\
& =\sum_{m=0}^{\infty} \sum_{j=0}^{n-1}\left[X_{m+1, j} \frac{\Gamma(\gamma(m+1)+j+1)}{\Gamma(\gamma(m+1)+j+1-\alpha)}-B X_{m, j}\right] t^{\gamma m+j+\beta} .
\end{aligned}
$$

So, if we choose

$$
X_{m+1, j}=B X_{m, j} \frac{\Gamma(\gamma(m+1)+j+1-\alpha)}{\Gamma(\gamma(m+1)+j+1)}, \quad m=0,1, \ldots
$$

in (7), it is guarantee that $Y(t)$ defined by (3) will satisfy the random fractional differential equation $\left({ }^{C} D_{0^{+}}^{\alpha} Y\right)(t)-B t^{\beta} Y(t)=0$. Taking into account that $Y^{(j)}(0)=X_{0, j}=A_{j}$ for $j=0,1, \ldots, n-1$, the recursion for the sequence of coefficients $X_{m, j}$ gives

$$
X_{m, j}=B^{m} A_{j} G_{m, j}, \quad G_{m, j}:=\prod_{k=1}^{m} \frac{\Gamma(k \gamma+j+1-\alpha)}{\Gamma(k \gamma+j+1)}>0, \quad m \geq 0,
$$


Table 1: Some important families of random variables that satisfy hypothesis $\mathbf{H 1}$.

\begin{tabular}{lccc}
\hline Distribution & $p$ & $\mathcal{H}$ & $\eta$ \\
\hline \hline Bounded & 0 & 1 & 1 \\
\hline Gaussian $\sim \mathrm{N}\left(0 ; \sigma^{2}\right)$ & $1 / 2$ & $\sigma \sqrt{2}$ & $\sigma$ \\
\hline Exponential $\sim \operatorname{Exp}(\lambda)$ & 1 & $2 / \lambda$ & $\sqrt{2} / \lambda$ \\
\hline Weibull $\sim \operatorname{We}(a ; b)$ & $1 / b$ & $a(2 / b)^{1 / b}$ & $a \sqrt{\Gamma(1+2 / b)}$ \\
\hline
\end{tabular}

where as usual we implicitly assume that $\prod_{k=1}^{m} y_{k}=1$ when $m=0$. Therefore

$$
Y(t)=\sum_{j=0}^{n-1} Y_{j}(t)=\sum_{j=0}^{n-1}\left(\sum_{m=0}^{\infty} B^{m} A_{j} G_{m, j} t^{\gamma m+j}\right)
$$

is a mean square solution of (1), provided that, for each $j$, the stochastic processes $Y_{j}(t)$ and $\frac{\mathrm{d}^{i} Y_{j}(t)}{\mathrm{d} t^{i}}$, $i=1,2, \ldots, n$ are mean square convergent and mean square uniformly convergent on $\mathcal{T}$, respectively. In order to justify that these conditions fulfil, we will assume the following hypotheses for the random inputs $A_{j}, j=0,1,2, \ldots$ and $B$ :

H1: There exist constants $\eta, \mathcal{H}>0$ and $p \geq 0$ and $m, m_{0}$ integers such that

$$
\left\|B^{m}\right\|_{2} \leq \eta \mathcal{H}^{m-1}((m-1) !)^{p}, \quad \forall m: m \geq m_{0} \geq 1 .
$$

H2: For every $j, j=0,1, \ldots, n-1, A_{j}$ and $B$ are independent random variables.

In Table 1, we collect some relevant families of random variables that satisfy hypothesis $\mathbf{H 1}$ by indicating the value of involved parameters $p, \mathcal{H}$ and $\eta$. In particular, it is important to point out that any bounded random variable satisfies H1, so in practice, any unbounded random variable can be adequately truncated so that the truncated (hence bounded) random variable behaves approximately as the original unbounded random variable, that is, approximately preserving its main probabilistic information like mean, variance, etc. A way to do that, which is supported by Markov-Chebyshev type inequalities, is to consider an interval with center the mean and radius a multiple, say $k>0$, of the standard deviation of the unbounded random variable [30, Ch. 5]. Taking $k$ large enough, most of the probability mass is captured and the mean and standard deviation of the original unbounded random variable are then approximated. Alternatively, one can construct a truncated parametric distribution preserving a number of statistical moments (mean, variance, etc.) so that the new (bounded) distribution approximates quite well the original unbounded distribution, this approach is based upon the matching moment method [30, Ch. 5].

In the numerical examples that will be shown in Section 4 , the random coefficient $B$ will be chosen from the distributions listed in Table 1.

As a consequence of hypotheses $\mathbf{H 1}$ and $\mathbf{H} 2$, one gets

$$
\left\|B^{m} A_{j} G_{m, j} t^{\gamma m+j}\right\|_{2} \leq \eta \mathcal{H}^{m-1}((m-1) !)^{p}\left\|A_{j}\right\|_{2} G_{m, j} t^{\gamma m+j}:=\delta_{m, j}(t),
$$

for $j=0,1, \ldots, n-1, m \geq 1$ integer and $t>0$. For every $j$ fixed, we study the domain of convergence of the infinite sum $\sum_{m=0}^{\infty} \delta_{m, j}(t)$ by applying the ratio test. The approximation $\Gamma(x+1) \approx x^{x} \mathrm{e}^{-x} \sqrt{2 \pi x}$ as $x \rightarrow \infty$ will be used too. Recall that $\gamma=\alpha+\beta$. 


$$
\begin{aligned}
& \lim _{m \rightarrow \infty} \frac{\delta_{m+1, j}(t)}{\delta_{m, j}(t)} \\
& =\lim _{m \rightarrow \infty} \mathcal{H} m^{p} \frac{\Gamma((m+1) \gamma+j+1-\alpha)}{\Gamma((m+1) \gamma+j+1)} t^{\gamma} \\
& =\lim _{m \rightarrow \infty} \frac{\mathcal{H} m^{p}[(m+1) \gamma+j-\alpha]^{(m+1) \gamma+j-\alpha} \mathrm{e}^{-((m+1) \gamma+j-\alpha)} \sqrt{2 \pi((m+1) \gamma+j-\alpha)}}{[(m+1) \gamma+j]^{(m+1) \gamma+j} \mathrm{e}^{-((m+1) \gamma+j)} \sqrt{2 \pi((m+1) \gamma+j)}} t^{\gamma} \\
& =\lim _{m \rightarrow \infty} \mathcal{H} m^{p}\left[\frac{(m+1) \gamma+j-\alpha}{(m+1) \gamma+j}\right]^{(m+1) \gamma+j-\alpha}\left(\frac{1}{(m+1) \gamma+j}\right)^{\alpha} \\
& \times \mathrm{e}^{\alpha} \sqrt{\frac{(m+1) \gamma+j-\alpha}{(m+1) \gamma+j}} t^{\alpha+\beta} \\
& =\lim _{m \rightarrow \infty} \mathcal{H} m^{p}\left[\frac{(m+1) \gamma+j-\alpha}{(m+1) \gamma+j}\right]^{m \gamma}\left[\frac{(m+1) \gamma+j-\alpha}{(m+1) \gamma+j}\right]^{\beta+j}\left(\frac{1}{(m+1) \gamma+j}\right)^{\alpha} \\
& \times \mathrm{e}^{\alpha} \sqrt{\frac{(m+1) \gamma+j-\alpha}{(m+1) \gamma+j}} t^{\alpha+\beta} .
\end{aligned}
$$

Now taking into account that $\lim _{m \rightarrow+\infty}\left[\frac{(m+1) \gamma+j-\alpha}{(m+1) \gamma+j}\right]^{m \gamma}=\mathrm{e}^{-\alpha}$ and $\lim _{m \rightarrow+\infty} \frac{(m+1) \gamma+j-\alpha}{(m+1) \gamma+j}=1$, for $t \geq 0$ one gets

$$
\lim _{m \rightarrow+\infty} \frac{\delta_{m+1}(t)}{\delta_{m}(t)}=t^{\alpha+\beta} \mathcal{H} \lim _{m \rightarrow+\infty} \frac{m^{p}}{((m+1)(\alpha+\beta)+j)^{\alpha}}=\left\{\begin{array}{cll}
0 & \text { if } & 0 \leq p<\alpha, \\
\mathcal{H} t^{\beta}\left(\frac{t}{\alpha+\beta}\right)^{\alpha} & \text { if } & p=\alpha .
\end{array}\right.
$$

So, we have proved that the majorizing series $\sum_{m=0}^{\infty} \delta_{m, j}(t)$ converges for every $t$ in $\mathcal{D}$, where

$$
\mathcal{D}=\left\{\begin{array}{cll}
{[0, \infty)} & \text { if } & 0 \leq p<\alpha \\
{\left[0, \frac{(\alpha+\beta)^{\frac{\alpha}{\alpha+\beta}}}{\mathcal{H}^{\frac{1}{\alpha+\beta}}}\left[\begin{array}{ll}
\text { if } & p=\alpha
\end{array}\right.\right.} &
\end{array}\right.
$$

Summarizing, we have found sufficient conditions for the second-order stochastic process $Y(t)$ to be a mean square solution of the IVP given in (1). The next result states our findings.

Theorem 1. If the random variables $A_{j}, j=0,1, \ldots, n-1$, and B satisfy conditions $\boldsymbol{H} \mathbf{1}$ and $\boldsymbol{H} 2$, then the stochastic process

$$
Y(t)=\sum_{j=0}^{n-1}\left(\sum_{m=0}^{\infty} B^{m} A_{j} G_{m, j} t^{\gamma m+j}\right), \quad \gamma=\alpha+\beta,
$$

where

$$
G_{m, j}=\prod_{k=1}^{m} \frac{\Gamma(k \gamma+j+1-\alpha)}{\Gamma(k \gamma+j+1)}, \quad m=0,1,2, \ldots,
$$

is a mean square solution of problem (1) for $t \in \mathcal{T}=\left[t_{1}, t_{2}\right] \subset \mathcal{D} \subset \mathbb{R}$, being $\mathcal{D}$ given in (9).

Notice that the domain of convergence of $Y(t)$ depends on the relation between the order of the fractional derivative, $\alpha>0$, and the value of $p$, involved in the hypothesis $\mathbf{H 1}$, which only affects the random coefficient $B$. 


\section{Probabilistic properties of the solution stochastic process}

Once we have constructed a solution stochastic process, $Y(t)$, to the random fractional IVP (1), an important issue is to compute its main statistical properties, such as the mean $(\mathbb{E}[Y(t)])$, variance $(\mathbb{V}[Y(t)])$ and covariance $(\mathbb{C o v}[Y(t), Y(s)])$ functions. Since the solution stochastic process, $Y(t)$, has been constructed via the infinite series (10)-(11), it must be truncated to keep feasible the computational burden. Thus, for a positive integer $M$, hereinafter we will consider the truncated series, $Y_{M}(t)$, of $Y(t)$, defined by

$$
Y_{M}(t)=\sum_{j=0}^{n-1}\left(\sum_{m=0}^{M} B^{m} A_{j} G_{m, j} t^{\gamma m+j}\right)
$$

To this end, the following result will play a key role and it also justifies why we have made the decision to conduct our study of uncertainty in the random fractional IVP (1) using the mean square convergence instead of considering other stochastic convergences like almost surely, in probability or in distribution.

Proposition 1. [10, Th. 4.3.1] Let $\left\{X_{n}: n \geq 0\right\}$ be a mean square convergent sequence of random variables in $\mathrm{L}^{2}(\Omega)$ and let us denote its limit by $X \in \mathrm{L}^{2}(\Omega)$ as $n \rightarrow \infty$. Then,

$$
\mathbb{E}\left[X_{n}\right] \underset{n \rightarrow+\infty}{\longrightarrow} \mathbb{E}[X], \quad \mathbb{V}\left[X_{n}\right] \underset{n \rightarrow+\infty}{\longrightarrow} \mathbb{V}[X]
$$

If, we further assume that $\left\{Y_{m}: m \geq 0\right\}$ is a mean square convergent sequence of random variables in $\mathrm{L}^{2}(\Omega)$, being $Y \in \mathrm{L}^{2}(\Omega)$ its limit as $m \rightarrow \infty$, then

$$
\begin{gathered}
\mathbb{E}\left[X_{n} Y_{m}\right] \underset{n, m \rightarrow+\infty}{\longrightarrow} \mathbb{E}[X Y], \\
\operatorname{Cov}\left[X_{n}, Y_{m}\right] \underset{n, m \rightarrow+\infty}{\longrightarrow} \mathbb{C o v}[X, Y] .
\end{gathered}
$$

Notice that we can take advantage of this result because mean square convergence of the infinite series defining $Y(t)$ for $t>0$ has been rigorously established in Theorem 1.

Taking the expectation operator in (12) and using its linearity and hypothesis $\mathbf{H 2}$, it is clear that the mean of $Y_{M}(t)$ is given by

$$
\mathbb{E}\left[Y_{M}(t)\right]=\sum_{j=0}^{n-1}\left(\sum_{m=0}^{M} \mathbb{E}\left[B^{m}\right] \mathbb{E}\left[A_{j}\right] G_{m, j} t^{\gamma m+j}\right) .
$$

Notice that in order to compute approximations of the expectation to the solution stochastic process $Y(t)$ of the random IVP (1) via $Y_{M}(t)$, the expectation of the initial conditions, $A_{j}, 0 \leq$ $j \leq n-1$, and the moments with respect to (w.r.t.) the origin up to order $M$ of the random coefficient $B$ are just required.

Now, we are interested in computing an approximation of the variance of $Y_{M}(t)$. To this end, and for the sake of generality, we first calculate its cross-covariance 


$$
\begin{aligned}
\mathbb{C}_{Y_{M}, Y_{N}}(t, s) & :=\operatorname{Cov}\left[Y_{M}(t), Y_{N}(s)\right] \\
& =\sum_{j=0}^{n-1} \sum_{k=0}^{n-1} \operatorname{Cov}\left[\sum_{r=0}^{M} B^{r} A_{j} G_{r, j} t^{\gamma r+j}, \sum_{m=0}^{N} B^{m} A_{k} G_{m, k} s^{\gamma m+k}\right] \\
& =\sum_{j=0}^{n-1} \sum_{k=0}^{n-1} \sum_{r=0}^{M} \sum_{m=0}^{N} \mathbb{C o v}\left[B^{r} A_{j}, B^{m} A_{k}\right] G_{r, j} G_{m, k} t^{\gamma r+j} s^{\gamma m+k} \\
& =\sum_{j=0}^{n-1} \sum_{k=0}^{n-1} \sum_{r=0}^{N} \sum_{m=0}^{M}\left(\mathbb{E}\left[A_{j} A_{k} B^{m+r}\right]-\mathbb{E}\left[B^{r} A_{j}\right] \mathbb{E}\left[B^{m} A_{k}\right]\right) G_{r, j} G_{m, k} t^{\gamma r+j} s^{\gamma m+k} \\
& =\sum_{j=0}^{n-1} \sum_{k=0}^{n-1} \sum_{r=0}^{N} \sum_{m=0}^{M}\left(\mathbb{E}\left[A_{j}\right] \mathbb{E}\left[A_{k}\right] \mathbb{E}\left[B^{m+r}\right]-\mathbb{E}\left[A_{j}\right] \mathbb{E}\left[A_{k}\right] \mathbb{E}\left[B^{m}\right] \mathbb{E}\left[B^{r}\right]\right) G_{r, j} G_{m, k} t^{\gamma r+j} s^{\gamma m+k} \\
& =\sum_{j=0}^{n-1} \sum_{k=0}^{n-1} \sum_{r=0}^{N} \sum_{m=0}^{M} \mathbb{E}\left[A_{j}\right] \mathbb{E}\left[A_{k}\right]\left(\mathbb{E}\left[B^{m+r}\right]-\mathbb{E}\left[B^{m}\right] \mathbb{E}\left[B^{r}\right]\right) G_{r, j} G_{m, k} t^{\gamma r+j} s^{\gamma m+k} \\
& =\sum_{j=0}^{n-1} \sum_{k=0}^{n-1} \sum_{r=0}^{N} \sum_{m=0}^{M} \mathbb{E}\left[A_{j}\right] \mathbb{E}\left[A_{k}\right] \mathbb{C o v}\left[B^{m}, B^{r}\right] G_{r, j} G_{m, k} t^{\gamma r+j} s^{\gamma m+k},
\end{aligned}
$$

where we have used the bilinearity of the covariance, its definition and the (mutually) independence of random variables $A_{0}, A_{1}, \ldots, B$, assumed in hypothesis $\mathbf{H 2}$. In the particular case that $M=N$ and $t=s$ in (14), one obtains the variance of the $Y_{M}(t)$

$$
\begin{aligned}
\mathbb{V}\left[Y_{M}(t)\right] & =\operatorname{Cov}\left[Y_{M}(t), Y_{M}(t)\right] \\
& =\sum_{j=0}^{n-1} \sum_{k=0}^{n-1} \sum_{r=0}^{M} \sum_{m=0}^{M} \mathbb{E}\left[A_{j}\right] \mathbb{E}\left[A_{k}\right] \operatorname{Cov}\left[B^{m}, B^{r}\right] G_{r, j} G_{m, k} t^{\gamma r+j} s^{\gamma m+k} \\
& =\sum_{j=0}^{n-1} \sum_{k=0}^{n-1} \sum_{r=0}^{M} \sum_{m=0}^{M} \mathbb{E}\left[A_{j}\right] \mathbb{E}\left[A_{k}\right]\left(\mathbb{E}\left[B^{m+r}\right]-\mathbb{E}\left[B^{m}\right] \mathbb{E}\left[B^{r}\right]\right) G_{r, j} G_{m, k} t^{\gamma r+j} s^{\gamma m+k}
\end{aligned}
$$

Notice that in order to compute approximations of the variance to the solution stochastic process $Y(t)$ of the random IVP (1) via $Y_{M}(t)$, the expectation of the initial conditions, $A_{j}, 0 \leq$ $j \leq n-1$, as well as the moments w.r.t. the origin up to order $2 M$ of the random coefficient $B$ are just required.

Remark 1. Now, we make a constructive comment related to hypothesis H2. In Section 2, to prove mean square convergence of the solution stochastic process $Y(t)$ given by (10), hypothesis $\mathbf{H} 2$ could be relaxed assuming that $A_{j}$ and $B$ are independent random variables for every $j: 0 \leq$ $j \leq n-1$ instead (to see this, just check inequality (8) and apply [31, Th. 3, p. 92]). However, to keep a common assumption throughout the whole presentation of the manuscript we have embraced (mutually) independence among all involved random variables in $\mathbf{H 2}$, which is used to give a handy expression for the covariance, and thus for the variance too (see expressions (14) and (15), respectively). 
Remark 2. In the proof of Th. 1 , the set $\mathcal{D}$ is the interval where the deterministic majorizing series, $\sum_{m=0}^{\infty} \delta_{m, j}(t)$, for the solution $Y(t)$ is convergent. As a consequence, the solution $Y(t)$ is mean square convergent on $\mathcal{D}$, since $Y(t)$ was majorized by $\sum_{m=0}^{\infty} \delta_{m, j}(t)$ with the norm $\|\cdot\|_{2}$. However, the interval of convergence of $Y(t)$ may be larger than $\mathcal{D}$. As a result of Proposition 1 , the above approximations of the mean, $\mathbb{E}\left[Y_{M}(t)\right]$, and the variance, $\mathbb{V}\left[Y_{M}(t)\right]$, of $Y(t)$ are also convergent on $\mathcal{D}$. As in the case that $p=\alpha$, the interval $\mathcal{D}$ is bounded, $\mathcal{D}=\left[0, \frac{(\alpha+\beta)^{\frac{\alpha}{\alpha+\beta}}}{\mathcal{H}^{\frac{1}{\alpha+\beta}}}[\right.$, the approximations of the variance (and thus of the mean) may converge in larger intervals. This issue will be illustrated later in Example 3.

\section{Numerical examples}

This section is addressed to present some numerical examples where our previous theoretical findings are illustrated. In all the following examples, we will compute approximations to the mean $\left(\mathbb{E}\left[Y_{M}(t)\right]\right)$ and the variance/standard deviation $\left(\mathbb{V}\left[Y_{M}(t)\right] / \sigma\left[Y_{M}(t)\right]\right)$ of the solution stochastic process at different time instants $t$ by increasing the order of truncation $M$ using the expressions (13) and (15), respectively. The results are presented via graphical representations or numerical tables of these statistical moments. In some of the examples, we have also calculated the relative error of consecutive approximations of these two statistical moments with respect to the order of truncation

$$
\begin{gathered}
\operatorname{RE}(\text { Mean })(t ; M)=\left|\frac{\mathbb{E}\left[Y_{M+1}(t)\right]-\mathbb{E}\left[Y_{M}(t)\right]}{\mathbb{E}\left[Y_{M}(t)\right]}\right|, \\
\operatorname{RE}(\text { Variance })(t ; M)=\left|\frac{\mathbb{V}\left[Y_{M+1}(t)\right]-\mathbb{V}\left[Y_{M}(t)\right]}{\mathbb{V}\left[Y_{M}(t)\right]}\right| .
\end{gathered}
$$

The examples are devised to illustrate the two possible situations with respect the domain of convergence established in Th. 1. Observe that both cases depend on the relationship between $\alpha$ (order of the fractional derivative) and $p$ (parameter involved in hypothesis $\mathbf{H 1}$ and related to the behaviour of moments w.r.t. the origin of random variable $B$ ). The case $p<\alpha$ is illustrated in the Example 2, while the case $p=\alpha$ is analysed in the Example 3. As the IVP (1) corresponds to the random classical Airy differential equation when $\alpha=2$ and $\beta=1$, we will begin with Example 1 where this equation is analysed taking values of parameter $\alpha$ such that $\alpha \rightarrow 2^{-}$. Finally, we point out that in these three examples we have considered a wide variety of probability distributions for the random coefficient $B$ and different numerical values for the moments of initial conditions $A_{j}$, with the aim of showing the generality when applying the theoretical results previously established. As required in hypothesis $\mathbf{H 2}$, in the following examples we will assume that $B$ and the involved initial conditions $A_{j}$ are independent random variables.

Example 1. In order to check that the our theoretical findings for the random Airy fractional differential equation are consistent with the corresponding ones established in the previous work [27] for the random Airy ordinary differential equation, we consider the random fractional IVP (1) with $\beta=1, B \sim B e(2,3)$ (Be $(2,3)$ stands for a Beta distribution) and the initial conditions $A_{0}$ and $A_{1}$, that are random variables such that

$$
\mathbb{E}\left[A_{0}\right]=1, \mathbb{E}\left[A_{0}^{2}\right]=2, \mathbb{E}\left[A_{1}\right]=2, \mathbb{E}\left[A_{1}^{2}\right]=5 .
$$

In Tables 2 and 3, we collect the values of the approximations of the mean and variance of the solution stochastic process computed via (13) and (15) with $M=10$, respectively. For ease of 


\begin{tabular}{l|cccccc}
\hline \hline Mean & $\alpha=1.8$ & $\alpha=1.85$ & $\alpha=1.9$ & $\alpha=1.95$ & $\alpha=1.99$ & $\alpha=1.999$ \\
\hline \hline$t=0.00$ & 1.0000 & 1.0000 & 1.00000 & 1.0000 & 1.0000 & 1.0000 \\
\hline$t=0.25$ & 1.4978 & 1.4981 & 1.4983 & 1.4985 & 1.4987 & 1.4987 \\
\hline$t=0.50$ & 1.9814 & 1.9831 & 1.9847 & 1.9862 & 1.9873 & 1.9875 \\
\hline$t=0.75$ & 2.4325 & 2.4377 & 2.4425 & 2.4469 & 2.4503 & 2.451 \\
\hline$t=1.00$ & 2.8289 & 2.8396 & 2.8498 & 2.8594 & 2.8668 & 2.8684 \\
\hline$t=1.25$ & 3.1461 & 3.1643 & 3.1818 & 3.1987 & 3.2117 & 3.2146 \\
\hline$t=1.50$ & 3.3603 & 3.3868 & 3.4128 & 3.4382 & 3.4582 & 3.4626 \\
\hline$t=1.75$ & 3.4513 & 3.4853 & 3.5192 & 3.5530 & 3.5799 & 3.5859 \\
\hline$t=2.00$ & 3.4064 & 3.4446 & 3.4835 & 3.5231 & 3.5553 & 3.5625 \\
\hline
\end{tabular}

Table 2: Values of the expectation of the solution stochastic process of the random fractional IVP (1) computed by (13) with $M=10$ in the context of Example 1 .

\begin{tabular}{c|cccccc}
\hline \hline Variance & $\alpha=1.8$ & $\alpha=1.85$ & $\alpha=1.9$ & $\alpha=1.95$ & $\alpha=1.99$ & $\alpha=1.999$ \\
\hline \hline$t=0.00$ & 1.0000 & 1.0000 & 1.0000 & 1.0000 & 1.0000 & 1.0000 \\
\hline$t=0.25$ & 1.0589 & 1.0593 & 1.0597 & 1.0601 & 1.0603 & 1.0603 \\
\hline$t=0.50$ & 1.2227 & 1.2252 & 1.2274 & 1.2295 & 1.2311 & 1.2314 \\
\hline$t=0.75$ & 1.4680 & 1.4748 & 1.4811 & 1.4870 & 1.4915 & 1.4925 \\
\hline$t=1.00$ & 1.7639 & 1.7772 & 1.7900 & 1.8022 & 1.8116 & 1.8137 \\
\hline$t=1.25$ & 2.0769 & 2.0980 & 2.1187 & 2.1389 & 2.1548 & 2.1583 \\
\hline$t=1.50$ & 2.3820 & 2.4101 & 2.4381 & 2.4660 & 2.4882 & 2.4932 \\
\hline$t=1.75$ & 2.6755 & 2.7074 & 2.7398 & 2.7725 & 2.7990 & 2.8049 \\
\hline$t=2.00$ & 2.9803 & 3.0135 & 3.0470 & 3.0809 & 3.1084 & 3.1146 \\
\hline
\end{tabular}

Table 3: Values of the variance of the solution stochastic process to the random fractional IVP (1) computed by (15) with $M=10$ in the context of Example 1 .

comparison, the values of the inputs as well as the time instants where the mean and the variance of the solution have been computed, are the same as in Example 12 of [27]. In Tables 2 and 3 the values of $\alpha$ parameter are taken numbers such as $\alpha \rightarrow 2^{-}$. We evince rapid convergence for both the mean and the variance.

To complete our numerical experiment, in Figure 1 we have plotted the approximations of the mean (left) and the variance (right) for different order of truncations $M$ over the time interval $0 \leq t \leq 5$. From both plots, we can visualize the convergence of both statistical moments as $M$ increases over the whole time domain in agreement with our theoretical results. Notice that we have used greater values of $M$ for the variance $(M=7,8, \ldots, 11)$ than for the mean $(M=4,5, \ldots, 8)$ to get good approximations. This is an expected fact since the variance is a higher statistical moment than the mean.

To demonstrate the validity of the approximations for larger time intervals, in Figure 2 we have represented the approximations of both the mean and the variance in the interval $0 \leq t \leq 9$. Notice that to compute reliable approximations on this larger time interval, greater values of the truncation order $M$ have been required. Specifically, for the mean, we have taken $M=$ $18,19, \ldots, 22$, and for the variance, $M=21,22, \ldots, 25$. In Tables 4 and 5 we show the relative errors for the approximations of the mean and the variance, respectively, at the time instants $t=0,1, \ldots, 9$. As expected, we can observe that for $t$ fixed, these approximations improve as $M$ 

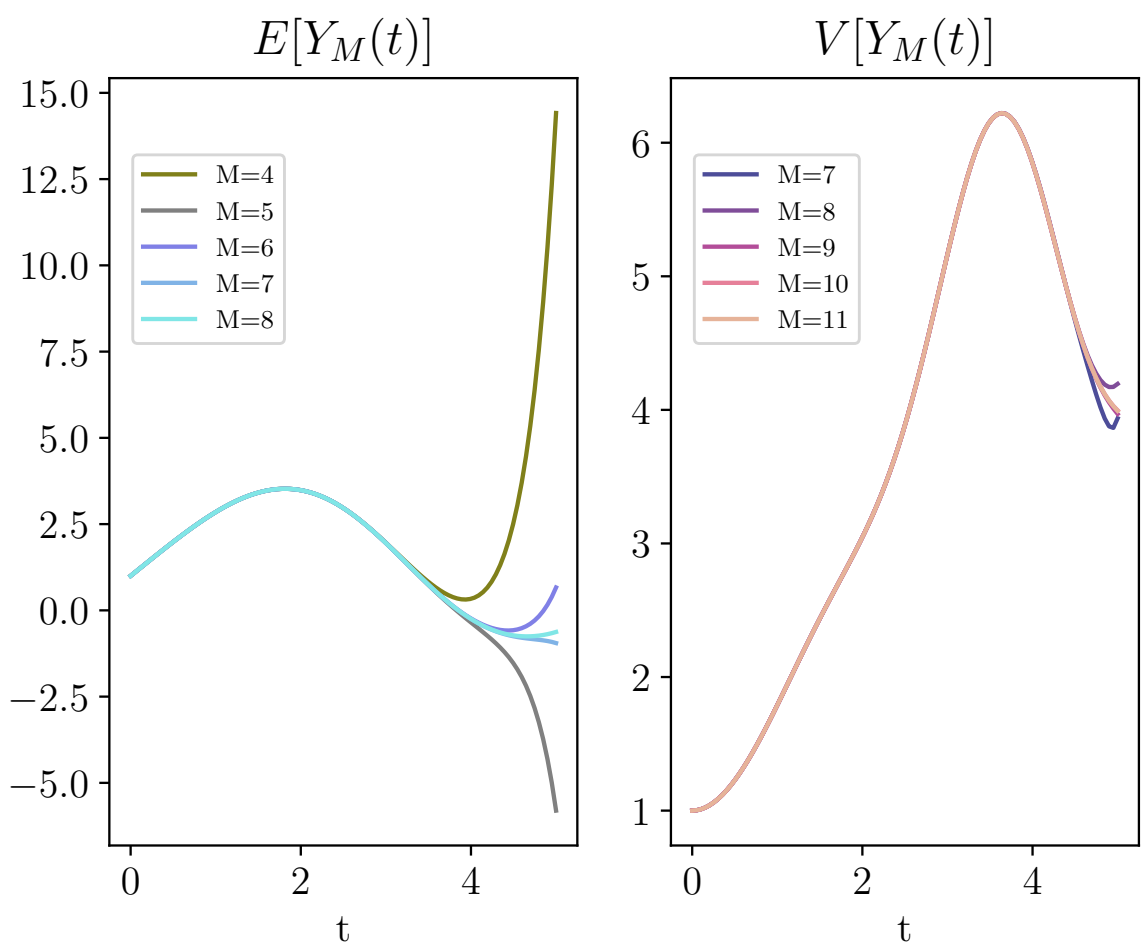

Figure 1: Approximations of the mean $\left(\mathbb{E}\left[Y_{M}(t)\right]\right)$ and the variance $\left(\mathbb{V}\left[Y_{M}(t)\right]\right)$ of the solution stochastic process to the random IVP (1) using different orders of truncations $M$ over the interval $t \in[0,5]$ in the context of Example 1. 


\begin{tabular}{c|cccccccccc}
\hline \hline RE(Mean) $(t ; M)$ & $t=0$ & $t=1$ & $t=2$ & $t=3$ & $t=4$ & $t=5$ & $t=6$ & $t=7$ & $t=8$ & $t=9$ \\
\hline \hline$M=18$ & 0.0 & 0.0 & 0.0 & 0.0 & 0.0 & 0.0 & 0.0 & 0.0385 & 2.0194 & 1.2306 \\
\hline$M=19$ & 0.0 & 0.0 & 0.0 & 0.0 & 0.0 & 0.0 & 0.0 & 0.0041 & 0.3200 & 1.2122 \\
\hline$M=20$ & 0.0 & 0.0 & 0.0 & 0.0 & 0.0 & 0.0 & 0.0 & 0.0004 & 0.0697 & 1.1899 \\
\hline$M=21$ & 0.0 & 0.0 & 0.0 & 0.0 & 0.0 & 0.0 & 0.0 & 0.0 & 0.0089 & 1.2018 \\
\hline$M=22$ & 0.0 & 0.0 & 0.0 & 0.0 & 0.0 & 0.0 & 0.0 & 0.0 & 0.0011 & 1.0551 \\
\hline$M=23$ & 0.0 & 0.0 & 0.0 & 0.0 & 0.0 & 0.0 & 0.0 & 0.0 & 0.0001 & 3.1418 \\
\hline$M=24$ & 0.0 & 0.0 & 0.0 & 0.0 & 0.0 & 0.0 & 0.0 & 0.0 & 0.0 & 0.2239 \\
\hline$M=25$ & 0.0 & 0.0 & 0.0 & 0.0 & 0.0 & 0.0 & 0.0 & 0.0 & 0.0 & 0.0410 \\
\hline
\end{tabular}

Table 4: Relative error for the mean, given by (16), at different time instants for different orders of truncation $M$ in the context of Example 1.

\begin{tabular}{c|cccccccccc}
\hline \hline RE(Variance) $(t ; M)$ & $t=0$ & $t=1$ & $t=2$ & $t=3$ & $t=4$ & $t=5$ & $t=6$ & $t=7$ & $t=8$ & $t=9$ \\
\hline \hline$M=18$ & 0.0 & 0.0 & 0.0 & 0.0 & 0.0 & 0.0 & 0.0 & 0.0 & 0.1519 & 0.9392 \\
\hline$M=19$ & 0.0 & 0.0 & 0.0 & 0.0 & 0.0 & 0.0 & 0.0 & 0.0 & 0.0002 & 0.9494 \\
\hline$M=20$ & 0.0 & 0.0 & 0.0 & 0.0 & 0.0 & 0.0 & 0.0 & 0.0 & 0.0007 & 0.9533 \\
\hline$M=21$ & 0.0 & 0.0 & 0.0 & 0.0 & 0.0 & 0.0 & 0.0 & 0.0 & 0.0001 & 0.8936 \\
\hline$M=22$ & 0.0 & 0.0 & 0.0 & 0.0 & 0.0 & 0.0 & 0.0 & 0.0 & 0.0 & 0.2753 \\
\hline$M=23$ & 0.0 & 0.0 & 0.0 & 0.0 & 0.0 & 0.0 & 0.0 & 0.0 & 0.0 & 0.0180 \\
\hline$M=24$ & 0.0 & 0.0 & 0.0 & 0.0 & 0.0 & 0.0 & 0.0 & 0.0 & 0.0 & 0.0006 \\
\hline$M=25$ & 0.0 & 0.0 & 0.0 & 0.0 & 0.0 & 0.0 & 0.0 & 0.0 & 0.0 & 0.0002 \\
\hline
\end{tabular}

Table 5: Relative error for the variance, given by (17), at different time instants for different orders of truncation $M$ in the context of Example 1.

increases.

Example 2. In this example we illustrate the case where $p<\alpha$. To this end, we assume that coefficient $B$ in the random IVP (1) has an exponential distribution with mean 4 , i.e. $B \sim \operatorname{Exp}(4)$, thus $p=1$ (see Table 1) and $\alpha=2.6$. We will take $\beta=1.7$ and the two first statistical moments w.r.t. the origin of the three random initial conditions are assumed to be

$$
\mathbb{E}\left[A_{0}\right]=1, \mathbb{E}\left[A_{0}^{2}\right]=2, \mathbb{E}\left[A_{1}\right]=2, \mathbb{E}\left[A_{1}^{2}\right]=5, \mathbb{E}\left[A_{2}\right]=4, \mathbb{E}\left[A_{2}^{2}\right]=20 .
$$

In Figure 3, we show the approximations of the mean, $\mathbb{E}\left[Y_{M}(t)\right]$, and the standard deviation, $\sigma\left[Y_{M}(t)\right]$, for different orders of truncations $M=2,3, \ldots, 6$ in the interval $t \in[0,5]$. We observe convergence being faster for the mean than for the standard deviation, as expected. According to Th. 1 and Prop. 1, in this context convergence of the mean and the standard deviation (or equivalently, the variance) takes place for every $t$. Naturally, to get better approximations the order of truncation $M$ needs to be increased as $t$ departs from the origin, $t=0$, where the initial condition is set. To better assess the convergence, in Tables 6 and 7 we have collected the values for the relative errors of the mean and the variance. We can see that figures are in fully agreement with the previous comments.

To complete the graphical analysis in this example, in Figure 4 we show the surface of the 

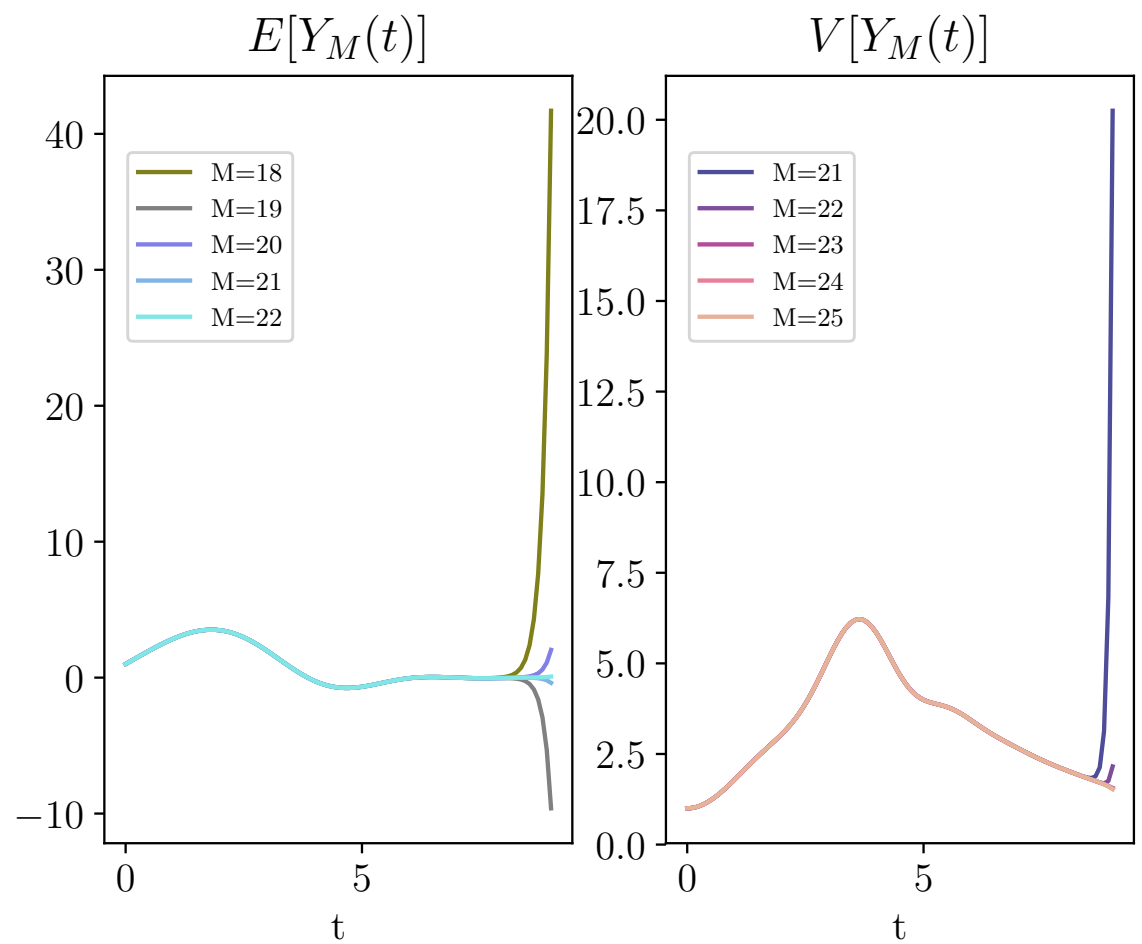

Figure 2: Approximations of the mean $\left(\mathbb{E}\left[Y_{M}(t)\right]\right)$ and the variance $\left(\mathbb{V}\left[Y_{M}(t)\right]\right)$ of the solution stochastic process to the random IVP (1) using different orders of truncations $M$ over the interval $t \in[0,9]$ in the context of Example 1.

\begin{tabular}{c|ccccccc}
\hline \hline RE(Mean) $(t ; M)$ & $t=1$ & $t=2$ & $t=3$ & $t=4$ & $t=5$ & $t=6$ & $t=7$ \\
\hline \hline$M=2$ & 0.0 & 0.0 & 0.0044 & 0.0781 & 0.4369 & 1.3189 & 2.9417 \\
\hline$M=3$ & 0.0 & 0.0 & 0.0003 & 0.0155 & 0.1674 & 0.6796 & 1.7185 \\
\hline$M=4$ & 0.0 & 0.0 & 0.0 & 0.0024 & 0.0569 & 0.3487 & 1.0506 \\
\hline$M=5$ & 0.0 & 0.0 & 0.0 & 0.0003 & 0.0163 & 0.1698 & 0.6493 \\
\hline$M=6$ & 0.0 & 0.0 & 0.0 & 0.0 & 0.0038 & 0.0756 & 0.3956 \\
\hline$M=7$ & 0.0 & 0.0 & 0.0 & 0.0 & 0.0007 & 0.0299 & 0.2326 \\
\hline$M=8$ & 0.0 & 0.0 & 0.0 & 0.0 & 0.0001 & 0.0103 & 0.1293 \\
\hline$M=9$ & 0.0 & 0.0 & 0.0 & 0.0 & 0.0 & 0.0031 & 0.0667 \\
\hline
\end{tabular}

Table 6: Relative error for the mean, given by (16), at different time instants for different orders of truncation $M$ in the context of Example 2. 


\begin{tabular}{c||ccccccc}
\hline \hline RE(Variance) $(t ; M)$ & $t=1$ & $t=2$ & $t=3$ & $t=4$ & $t=5$ & $t=6$ & $t=7$ \\
\hline$M=2$ & 0.0 & 0.0002 & 0.0427 & 0.6461 & 3.6097 & 14.6488 & 49.8305 \\
\hline \hline$M=3$ & 0.0 & 0.0 & 0.0037 & 0.1628 & 1.3583 & 6.1317 & 21.3876 \\
\hline$M=4$ & 0.0 & 0.0 & 0.0002 & 0.035 & 0.5557 & 3.0331 & 11.0941 \\
\hline$M=5$ & 0.0 & 0.0 & 0.0 & 0.0059 & 0.2195 & 1.6144 & 6.3768 \\
\hline$M=6$ & 0.0 & 0.0 & 0.0 & 0.0008 & 0.0775 & 0.8774 & 3.8925 \\
\hline$M=7$ & 0.0 & 0.0 & 0.0 & 0.0001 & 0.0232 & 0.4687 & 2.46 \\
\hline$M=8$ & 0.0 & 0.0 & 0.0 & 0.0 & 0.0058 & 0.2379 & 1.5805 \\
\hline$M=9$ & 0.0 & 0.0 & 0.0 & 0.0 & 0.0012 & 0.1113 & 1.0168 \\
\hline
\end{tabular}

Table 7: Relative error for the variance, given by (17), at different time instants for different orders of truncation $M$ in the context of Example 2.

correlation coefficient

$$
\rho_{Y_{M}}(t, s)=\frac{\mathbb{C}_{Y_{M}, Y_{M}}(t, s)}{\sigma_{Y_{M}}(t) \sigma_{Y_{M}}(s)},
$$

for $M=6$ (where numerical results are stabilized) over the domain $(t, s) \in[0,5] \times[0,5]$. Observe that the coefficient of correlation takes its highest value, namely 1 , when $t=s$ (on the diagonal of the domain) since in this case $Y_{M}(t)$ and $Y_{M}(s)$ are completely and positively correlated since both match. From this graphical representation and the statistical interpretation of the correlation coefficient, we observe that the linear dependence between random variables $Y_{M}(t)$ and $Y_{M}(s)$ decreases to 0 (uncorrelated) when $t \rightarrow 5$ and $s \rightarrow 0$ or vice versa, $t \rightarrow 0$ and $s \rightarrow 5$.

Example 3. This example is devised to illustrate the case the mean square convergence of the solution stochastic process (10)-(11) (hence its mean and its variance too) on a bounded interval, that is, when $p=\alpha$ (see Th. 1 and Prop. 1). In such a case, the interval of convergence is given by $\mathcal{D}=\left[0, \frac{(\alpha+\beta)^{\frac{\alpha}{\alpha+\beta}}}{\mathcal{H}^{\frac{1}{\alpha+\beta}}}[\right.$ (see Th. 1). To this end, we choose $\alpha=4.2$ and $B$ is a Weibull random variable of parameters $a=0.001$ and $b=1 / \alpha$ (see Table 1). So, according to Table 1 , $\mathcal{H}=a(2 / b)^{1 / b}=7.62$. As a consequence $\mathcal{D}=[0,2.56271[$. For the rest of input parameters of the random IVP (1), we take $\beta=1$ and

$$
\begin{gathered}
\mathbb{E}\left[A_{0}\right]=0, \mathbb{E}\left[A_{0}^{2}\right]=2, \mathbb{E}\left[A_{1}\right]=1, \mathbb{E}\left[A_{1}^{2}\right]=5, \mathbb{E}\left[A_{2}\right]=4, \mathbb{E}\left[A_{2}^{2}\right]=17 . \\
\mathbb{E}\left[A_{3}\right]=0.5, \mathbb{E}\left[A_{3}^{2}\right]=0.26, \mathbb{E}\left[A_{4}\right]=0.75, \mathbb{E}\left[A_{4}^{2}\right]=0.57
\end{gathered}
$$

In order to check that convergence of the mean and the variance in the interval $\mathcal{D}=\left[0, \frac{(\alpha+\beta)^{\frac{\alpha}{\alpha+\beta}}}{\mathcal{H}^{\frac{1}{\alpha+\beta}}}[\right.$, in Tables 8 and 9, we have computed the relative errors of consecutive approximations of both statistical moments (see expressions (16) and (17)), respectively. From the figures shown in these tables we numerically evince convergence as $M$ increases for $t \in \mathcal{D}$. To highlight the numerical behaviour of these relative errors about the value upper of the convergence interval, i.e. $t=2.56271$, we have included in Table 8 and Table 9 the values corresponding to times $t=2.4,2.5,2.6,2.7$. Observe that relative errors also decrease beyond $t=2.56271$ illustrating the comments raised in Remark 2. 

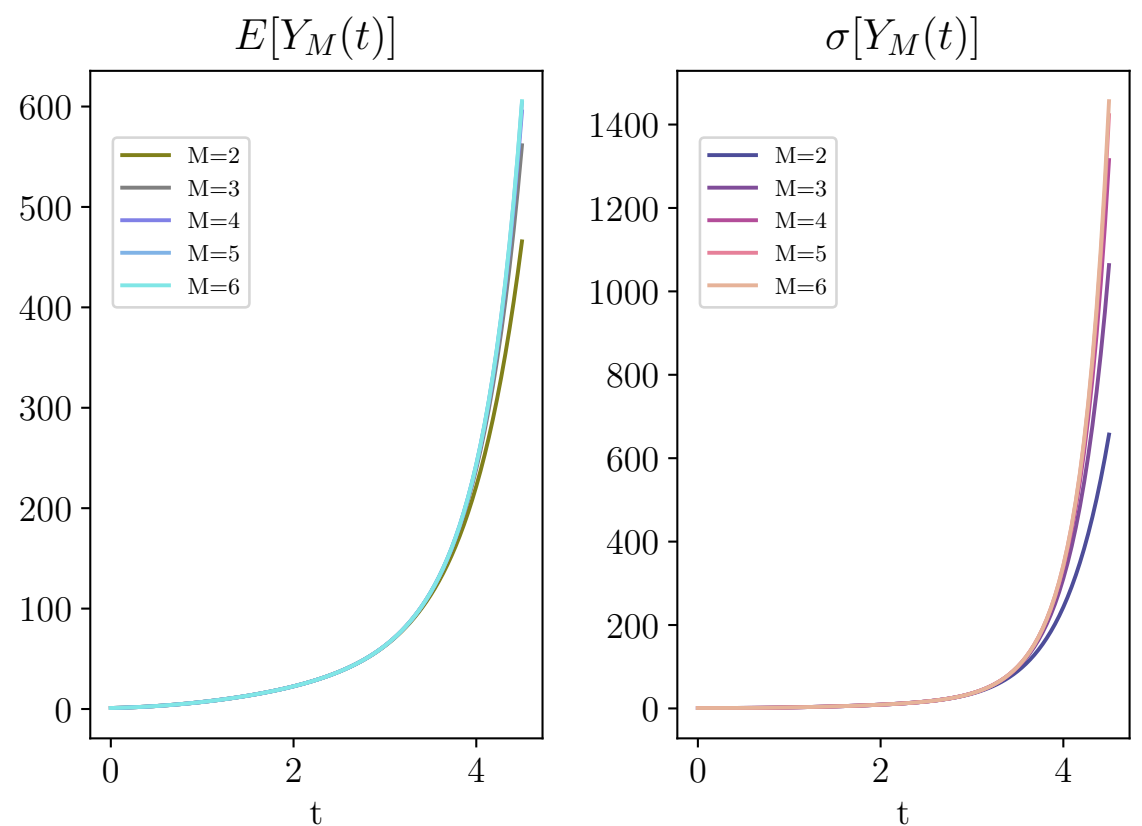

Figure 3: Approximations of the mean $\left(\mathbb{E}\left[Y_{M}(t)\right]\right)$ and the standard deviation $\left(\sigma\left[Y_{M}(t)\right]\right)$ of the solution stochastic process to the random IVP (1) using different orders of truncations $M$ in the interval $t \in[0,5]$ in the context of Example 2.

\begin{tabular}{c|ccc|cccc}
\hline \hline RE(Mean) $(t ; M)$ & $t=1$ & $t=2$ & $t=3$ & $t=2.4$ & $t=2.5$ & $t=2.6$ & $t=2.7$ \\
\hline \hline$M=2$ & 0.0 & 0.00119 & 0.10665 & 0.01083 & 0.01704 & 0.02598 & 0.03844 \\
\hline$M=3$ & 0.0 & $3 e-05$ & 0.02084 & 0.00069 & 0.00134 & 0.00252 & 0.00452 \\
\hline$M=4$ & 0.0 & 0.0 & 0.00372 & $4 e-05$ & $9 e-05$ & 0.00021 & 0.00046 \\
\hline$M=5$ & 0.0 & 0.0 & 0.0006 & 0.0 & $1 e-05$ & $2 e-05$ & $4 e-05$ \\
\hline$M=6$ & 0.0 & 0.0 & $9 e-05$ & 0.0 & 0.0 & 0.0 & 0.0 \\
\hline$M=7$ & 0.0 & 0.0 & $1 e-05$ & 0.0 & 0.0 & 0.0 & 0.0 \\
\hline$M=8$ & 0.0 & 0.0 & 0.0 & 0.0 & 0.0 & 0.0 & 0.0 \\
\hline$M=9$ & 0.0 & 0.0 & 0.0 & 0.0 & 0.0 & 0.0 & 0.0 \\
\hline
\end{tabular}

Table 8: Relative error for the mean, given by (16), at different time instants for different orders of truncation $M$ in the context of Example 3. 


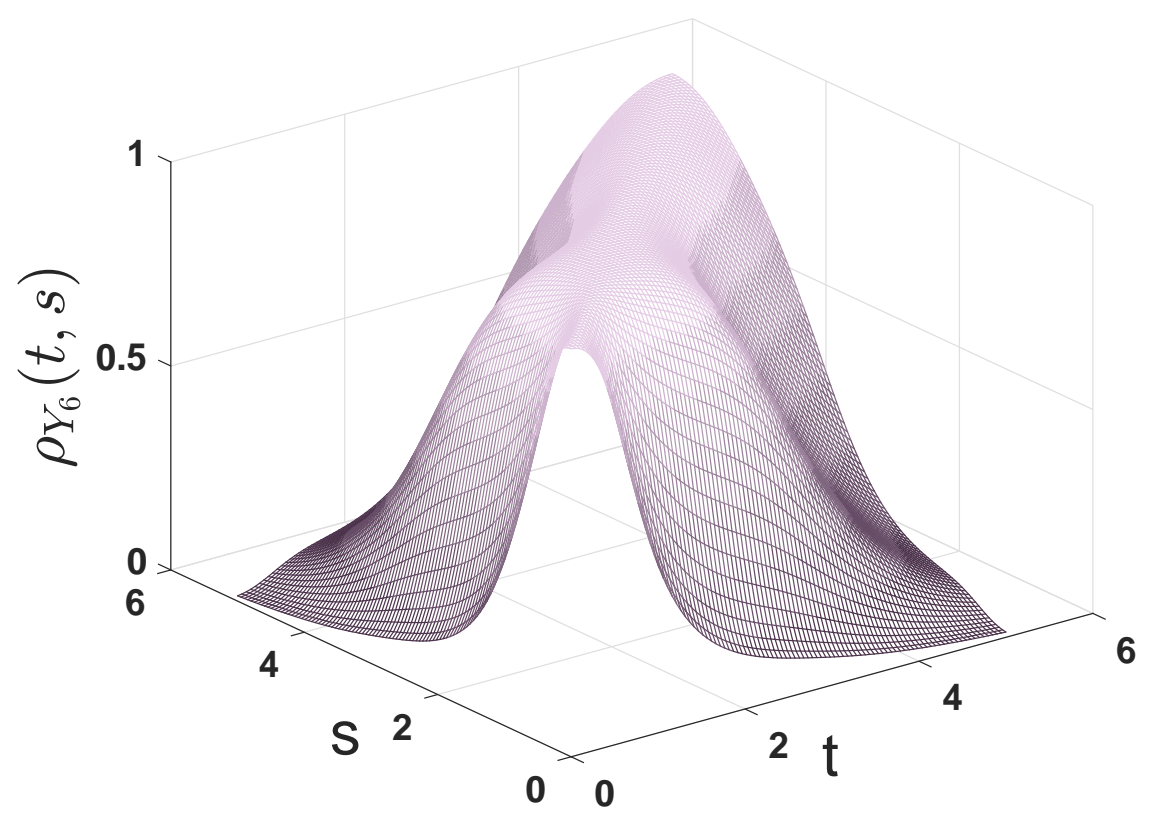

Figure 4: Surface of the correlation coefficient of the solution stochastic process, defined in (18), with $M=6$ over the domain $(t, s) \in[0,5] \times[0,5]$ in the context of Example 2 .

\begin{tabular}{c|ccc|cccc}
\hline \hline RE(Variance) $(t ; M)$ & $t=1$ & $t=2$ & $t=3$ & $t=2.4$ & $t=2.5$ & $t=2.6$ & $t=2.7$ \\
\hline \hline$M=2$ & 0.00018 & 0.80822 & 37.35409 & 4.61293 & 6.73642 & 9.6965 & 13.78835 \\
\hline$M=3$ & 0.0 & 0.27955 & 22.74314 & 2.45614 & 3.75086 & 5.57471 & 8.10991 \\
\hline$M=4$ & 0.0 & 0.10046 & 17.53194 & 1.6142 & 2.6038 & 4.01809 & 6.00033 \\
\hline$M=5$ & 0.0 & 0.03358 & 14.74921 & 1.15213 & 1.97189 & 3.16210 & 4.84565 \\
\hline$M=6$ & 0.0 & 0.01006 & 12.90896 & 0.85548 & 1.55995 & 2.59958 & 4.08308 \\
\hline$M=7$ & 0.0 & 0.0027 & 11.56632 & 0.64987 & 1.26903 & 2.19841 & 3.5352 \\
\hline$M=8$ & 0.0 & 0.00065 & 10.54187 & 0.50095 & 1.05405 & 1.89955 & 3.12451 \\
\hline$M=9$ & 0.0 & 0.00015 & 9.73999 & 0.38981 & 0.89008 & 1.67022 & 2.80801 \\
\hline
\end{tabular}

Table 9: Relative error for the variance, given by (17), at different time instants for different orders of truncation $M$ in the context of Example 3. 


\section{Approximating the probability density function via the Maximum Entropy Principle: An illustrative example}

So far we have computed approximations of the main statistical moments of the solution stochastic process $Y(t)$ given by (10), namely, the mean and the variance. In this section, we use this information to construct approximations of the probability density function (PDF), $f_{Y(t)}(y, t)$. The computation of the PDF is very useful since from it one can compute all one-dimensional statistical moments of $Y(t)$

$$
\mathbb{E}\left[(Y(t))^{r}\right]=\int_{\mathbb{R}} y^{r} f_{Y(t)}(y, t) \mathrm{d} y, \quad r=1,2, \ldots
$$

provided they exist. In this way a full probabilistic description of the solution stochastic process is obtained at every time instant $t$. In particular, the PDF $f_{Y(t)}(y, t)$ allows quantifying the probability that, for each $t$, the solution lies in a specific interval of interest, say $\left[y_{1}, y_{2}\right]$

$$
\mathbb{P}\left[y_{1} \leq Y(t) \leq y_{2}\right]=\int_{y_{1}}^{y_{2}} f_{Y(t)}(y, t) \mathrm{d} y
$$

which can be crucial in applications.

For these reasons this section is addressed to approximate $f_{Y(t)}(y, t)$ by calculating the PDF, $f_{Y_{M}(t)}(y, t)$, of the approximation $Y_{M}(t)$, given by (12). To achieve this goal, we will apply the Principle of Maximum Entropy (PME) taking advantage that we have obtained the mean, $\mathbb{E}\left[Y_{M}(t)\right]$, and the variance, $\mathbb{V}\left[Y_{M}(t)\right]$, of $Y_{M}(t)$ (see (13) and (15), respectively). Hereinafter, we first explain in detail the methodology and then we illustrate it by means of an example.

We first fix a time instant $t$ and an order of truncation $M$ large enough to guarantee that

$$
\mathbb{E}\left[Y_{M}(t)\right] \approx \mathbb{E}[Y(t)]=m_{1}(t) \equiv m_{1}, \quad \mathbb{E}\left[\left(Y_{M}(t)\right)^{2}\right] \approx \mathbb{E}\left[(Y(t))^{2}\right]=m_{2}(t) \equiv m_{2},
$$

i.e., that the approximations of the two first moments w.r.t to the origin (thus of the variance too) are good. Observe that this fact has been legitimated in Section 3 because of Proposition 1. Also notice that for convenience, in (20) we have hidden the time dependence in the notation for $m_{i}$, $i=1,2$. Then, we adapt the PME, that is typically applied within the context of random variables $[32,33,34]$, to our setting, i.e., we seek for a function $g=g(y)$, that will represent $f_{Y_{M}(t)}(y, t)$ for $t$ and $M$ fixed (and which approximates $f_{Y(t)}(y, t)$ ), that maximizes the following functional, usually referred to as Shannon's entropy,

$$
\mathcal{S}(g)=-\int_{a_{1}}^{a_{2}} g(y) \ln (g(y)) \mathrm{d} y,
$$

subject to the following constraints

$$
\int_{a_{1}}^{a_{2}} g(y) \mathrm{d} y=1, \quad \int_{a_{1}}^{a_{2}} y g(y) \mathrm{d} y=m_{1}, \quad \int_{a_{1}}^{a_{2}} y^{2} g(y) \mathrm{d} y=m_{2} .
$$

The first condition means that $g=g(y)$ (i.e., $\left.f_{Y_{M}(t)}(y, t)\right)$ is a PDF with support the interval $\left[a_{1}, a_{2}\right]$, while the two last conditions impose that $g=g(y)$ (i.e., $f_{Y_{M}(t)}(y, t)$ ) must satisfy the information related to the two first moments w.r.t. the origin. On the one hand, notice that according to Section 3 in our setting reliable approximations of both $m_{1}$ and $m_{2}$ are computable 
in practice. On the other hand, the support $\left[a_{1}, a_{2}\right]$ can be determined using the general approximating rule, derived from the Bienaymé-Chebyshev's inequality [35], which establishes that [mean \pm 10 standard deviation] contains the $99 \%$ of the total probability for any arbitrary random variable. In our case, this approximations will be applied to mean $=\mathbb{E}\left[Y_{M}(t)\right]$ and standard deviation $=\sqrt{\mathbb{V}\left[Y_{M}(t)\right]}$. We take advantage of this general approximation for the support $\left[a_{1}, a_{2}\right]$ because we do not know the true PDF, $f_{Y(t)}(y, t)$, of the solution stochastic process.

Therefore, in the admissible set

$$
\mathcal{A}=\left\{g:\left[a_{1}, a_{2}\right] \longrightarrow \mathbb{R}: \int_{a_{1}}^{a_{2}} g(y) \mathrm{d} y=1, \int_{a_{1}}^{a_{2}} y^{i} g(y) \mathrm{d} y=m_{i}, \quad i=1,2\right\},
$$

we seek for a function $g$ that maximizes the functional $\mathcal{S}$, defined in (21), subject to the three constraints given by (22). To determine the function $g$, we apply the variational version of Lagrange multiplier technique and then we consider the auxiliary Lagrangian function

$$
\begin{aligned}
\mathcal{L}\left(g, \lambda_{0}, \lambda_{1}, \lambda_{2}\right) & =\mathcal{S}(g)+\lambda_{0}\left(1-\int_{a_{1}}^{a_{2}} g(y) \mathrm{d} y\right)+\lambda_{1}\left(m_{1}-\int_{a_{1}}^{a_{2}} y g(y) \mathrm{d} y\right)+\lambda_{2}\left(m_{2}-\int_{a_{1}}^{a_{2}} y^{2} g(y) \mathrm{d} y\right) \\
& =-\int_{a_{!}}^{a_{2}} g(y)\left(\ln (g(y))+\sum_{i=0}^{2} \lambda_{i} y^{i}\right) \mathrm{d} y+\lambda_{0}+\lambda_{1} m_{1}+\lambda_{2} m_{2} .
\end{aligned}
$$

From variational calculus [36] we impose:

$$
\frac{\partial \mathcal{L}\left(g, \lambda_{0}, \lambda_{1}, \lambda_{2}\right)}{\partial g}=0, \quad \frac{\partial \mathcal{L}\left(g, \lambda_{0}, \lambda_{1}, \lambda_{2}\right)}{\partial \lambda_{i}}=0, \quad i=0,1,2 .
$$

The first condition leads to

$$
\frac{\partial \mathcal{L}\left(g, \lambda_{0}, \lambda_{1}, \lambda_{2}\right)}{\partial g}=-\int_{a_{1}}^{a_{2}}\left(\ln (g(y))+\sum_{i=0}^{2} \lambda_{i} y^{i}+1\right) \mathrm{d} y=0 .
$$

By imposing

$$
\ln (g(y))+\sum_{i=0}^{2} \lambda_{i} y^{i}+1=0, \quad a_{1} \leq y \leq a_{2},
$$

condition (24) is guaranteed. Now isolating $g=g(y)$, one gets

$$
g(y)=\mathbb{1}_{\left[a_{1}, a_{2}\right]} \mathrm{e}^{-1-\lambda_{0}-\lambda_{1} y-\lambda_{2} y^{2}},
$$

where $\mathbb{1}_{\left[a_{1}, a_{2}\right]}$ is the characteristic function on the interval $\left[a_{1}, a_{2}\right]$. The Lagrange multipliers $\lambda_{0}, \lambda_{1}$ and $\lambda_{2}$ are determined by using this expression (25) together with the conditions $\partial \mathcal{L}\left(g, \lambda_{0}, \lambda_{1}, \lambda_{2}\right) / \partial \lambda_{i}$, for $i=0,1,2$, given in (23). This leads to

$$
\int_{a_{1}}^{a_{2}} \mathrm{e}^{-1-\lambda_{0}-\lambda_{1} y-\lambda_{2} y^{2}} \mathrm{~d} y=1, \quad \int_{a_{1}}^{a_{2}} y \mathrm{e}^{-1-\lambda_{0}-\lambda_{1} y-\lambda_{2} y^{2}} \mathrm{~d} y=m_{1}, \quad \int_{a_{1}}^{a_{2}} y^{2} \mathrm{e}^{-1-\lambda_{0}-\lambda_{1} y-\lambda_{2} y^{2}} \mathrm{~d} y=m_{2} .
$$

This is a nonlinear system of equations involving the Gauss error function erf[·] when the integrals are calculated. Therefore, in practice numerical methods, such as Newton-Raphson, are required to determine its solution $\left(\lambda_{0}, \lambda_{1}, \lambda_{2}\right)$. Once these values have been computed, $g(y)$ is easily obtained by expression (25). 


\begin{tabular}{c|cccccc}
\hline \hline & $\lambda_{0}$ & $\lambda_{1}$ & $\lambda_{2}$ & $m_{1}=\mathbb{E}\left[Y_{30}(t)\right]$ & $m_{2}=\mathbb{E}\left[\left(Y_{30}(t)\right)^{2}\right]$ & $\mathbb{P}\left[y_{1} \leq Y(t) \leq y_{2}\right]$ \\
\hline \hline$t=0$ & 2.4189 & -1.0000 & 0.5000 & 1 & 2 & 0.686141 \\
\hline$t=1$ & 4.5155 & -1.5000 & 0.2500 & 3 & 11 & 0.684482 \\
\hline$t=2$ & 5.2236 & -1.0000 & 0.1000 & 5 & 30 & 0.684439 \\
\hline$t=3$ & 5.5202 & -0.7000 & 0.0500 & 7 & 59 & 0.683465 \\
\hline$t=4$ & 5.7179 & -0.5294 & 0.0290 & 9 & 98 & 0.683692 \\
\hline$t=5$ & 5.8749 & -0.4230 & 0.0190 & 11 & 147 & 0.681471 \\
\hline$t=6$ & 6.0082 & -0.3513 & 0.0135 & 13 & 206 & 0.683482 \\
\hline$t=7$ & 6.1250 & -0.3000 & 0.0100 & 15 & 275 & 0.681515 \\
\hline$t=8$ & 6.2292 & -0.2615 & 0.0076 & 17 & 354 & 0.679679 \\
\hline$t=9$ & 6.3235 & -0.2317 & 0.0060 & 19 & 443 & 0.682466 \\
\hline
\end{tabular}

Table 10: Values of $\lambda_{0}, \lambda_{1}, \lambda_{2}$ that solve the nonlinear system of equations (26) (with $m_{1}$ and $m_{2}$ given in the last two columns) and determine the PDF $g(y)$ (and thus $f_{Y_{M}(t)}(y, t)$ ) given by $(25)$ for $M=30$ at different times instants $t=0,1,2, \ldots, 9$. This function approximates the PDF $f_{Y(t)}(y, t)$ of the solution stochastic process to random fractional IVP (1) for the order of the fractional derivative $\alpha=1.9$ in the context of Example 1 .

To illustrate how to apply in practice this methodology, we have considered the same data as in the context of Example 1 to determine the PDF, $f_{Y_{M}(t)}(y, t)$, that approximates the PDF $f_{Y(t)}(y, t)$ of the solution stochastic process to random fractional IVP (1) for the order of the fractional derivative $\alpha=1.9$. To this end, we have fixed $t \in[0,9]$ and $M=30$ and using expressions (13) and (15), we have computed the moments $\mathbb{E}\left[Y_{M}(t)\right]$ and $\mathbb{E}\left[\left(Y_{M}(t)\right)^{2}\right]=\mathbb{V}\left[Y_{M}(t)\right]+\left(\mathbb{E}\left[Y_{M}(t)\right]\right)^{2}$. As we have previously pointed out, these values are good approximations of $m_{1}(t) \equiv m_{1}$ and $m_{2}(t) \equiv m_{2}$, respectively, which are required to set the system of nonlinear equations (26). Although the values $a_{1}$ and $a_{2}$ of the support are obviously different for each $t$, to simplify our exposition we must say that, according to Bienaymé-Chebyshev's inequality, is enough to take

$$
\left[a_{1}, a_{2}\right]=\left[\min _{t \in[0,9]} \mathbb{E}\left[Y_{M}(t)\right]-10 \sqrt{\mathbb{V}\left[Y_{M}(t)\right]}, \max _{t \in[0,9]} \mathbb{E}\left[Y_{M}(t)\right]+10 \sqrt{\mathbb{V}\left[Y_{M}(t)\right]}\right],
$$

for $M$ fixed (remember that in our case we have taken $M=30$ ). This leads to the following support $\left[a_{1}, a_{2}\right]=[-72,110]$. In order to calculate the $\operatorname{PDF} f_{Y_{M}(t)}(y, t)$ for $t \in[0,9]$, we have taken the time instants: $t=t_{k}=0.090 k, k=0,1, \ldots, 100$, and we have numerically computed the solutions $\left(\lambda_{0}, \lambda_{1}, \lambda_{2}\right)$ of the nonlinear system of equations (26). In Table 10, we show the values of $\lambda_{0}, \lambda_{1}, \lambda_{2}$ as well as of the moments $m_{1}$ and $m_{2}$ for $t=0,1, \ldots, 9$. To illustrate the usefulness of knowing the approximations of PDF $f_{Y_{M}(t)}(y, t)$, in the last column we have calculated the probability that the solution stochastic process lies in the interval $\left[y_{1}, y_{2}\right]$ for $t \in[0,9]$, by applying (19) with $\left.y_{1}=\mathbb{E}\left[Y_{M}(t)\right]-\sqrt{\mathbb{V}\left[Y_{M}(t)\right]}\right]$ and $\left.y_{2}=\mathbb{E}\left[Y_{M}(t)\right]+\sqrt{\mathbb{V}\left[Y_{M}(t)\right]}\right]$. Finally, in Figure 5 we have represented the $\operatorname{PDF} f_{Y_{M}(t)}(y, t)$ in the time interval $t \in[0,9]$ for $M=30$, and we have highlighted on the its surface the curves of the PDF corresponding to the time instants $t=0,1, \ldots, 9$.

\section{Conclusions}

In this paper we have studied an important class of random fractional differential equations, given by (1), that include as particular case the random Airy differential equation. We have constructed approximations of the solution stochastic process by means of random generalized 


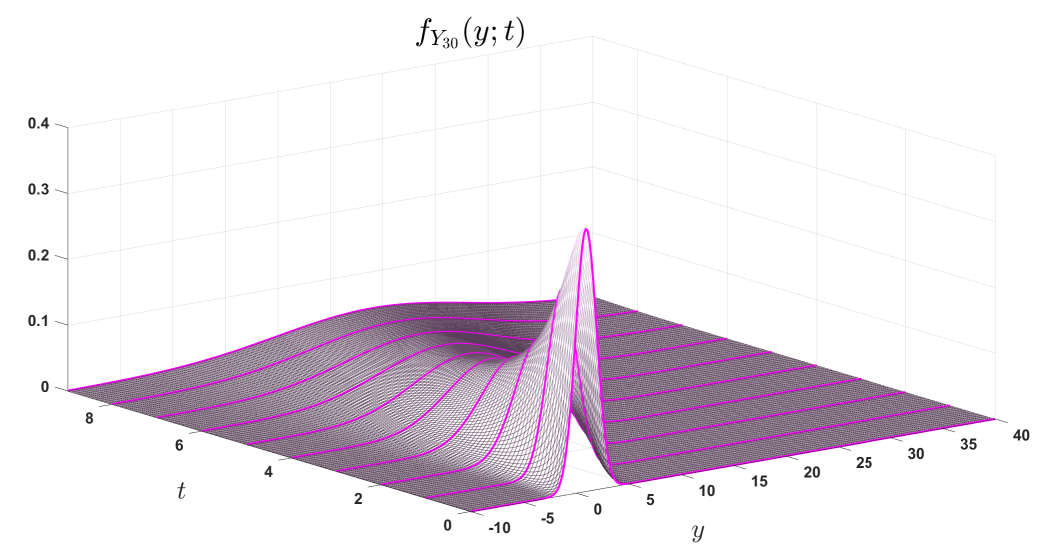

Figure 5: Approximate PDF, $f_{Y_{M}(t)}(y, t)$, of the solution stochastic process $Y(t)$ to the random fractional IVP (1) with order of the fractional derivative $\alpha=1.9$ in the context of Example 1 using the PME for the approximate solution stochastic process $Y_{M}(t)$ with $M=30$ and $0 \leq t \leq 9$.

power series and we have established sufficient conditions on random inputs (initial conditions and the coefficient $B$ ) in order to guarantee that random series is mean square convergent. The use of mean square convergence in our analysis is a key point since from its properties the convergence of the mean and the variance of the approximations to the corresponding exact ones are guaranteed. Furthermore, we have given explicit expressions to construct reliable approximations to the mean, the variance and the covariance functions of the solution. The study has relied heavily on the assumption made about the growth of statistical moments of the input coefficient $B$. We have shown that a wide range of important random variables satisfy such hypothesis. In future research, we will investigate alternative assumptions to $B$ in order to broaden the family of unbounded random variables that can play the role of input $B$. Nevertheless, an important issue of this paper is that we can approximate accurately unbounded random variables by truncating its domain so that the involved probabilistic error be as small as desired to get good approximations taking advantage of the results established here. Our numerical experiments evince the proposed method provides good approximations for the mean and the variance of the solution using a small order of truncation. Finally, we have taken advantage of the approximations of the mean and the variance of the solution stochastic process for the random fractional generalized Airy differential equation to construct reliable approximations of the probability density function of the solution. This is an important application of our approach since from the density function one can obtain a more comprehensive description of the solution via all its one-dimensional statistical moments and permits computing the probability that the solution lies in intervals of interest.

\section{Acknowledgements}

This work has been partially supported by the Ministerio de Economía y Competitividad grant MTM2017-89664-P.

The authors express their deepest thanks and respect to the editors and reviewers for their valuable comments. 


\section{Conflict of Interest Statement}

The authors declare that there is no conflict of interests regarding the publication of this article.

\section{References}

\section{References}

[1] I. Podlubny, Fractional Differential Equations: An Introduction to Fractional Derivatives, Fractional Differential Equations, to Methods of Their Solution and Some of Their Applications, Academic Press, London, 1998.

[2] A. A. Kilbas, H. M. Srivastava, J. J. Trujillo, Theory and Applications of Fractional Differential Equations, Elsevier Science, The Netherlands, 2006.

[3] S. B. Yuste, L. Acedo, An explicit finite difference method and a new von Neumann-type stability analysis for fractional diffusion equations, SIAM Journal of Numerical Analysis 42 (5) (2005) 1862-1874. doi:10.1137/030602666.

[4] Y. Khan, Q. Wu, N. Faraz, A. Yildirim, M. Madani, New fractional analytical approach via a modified RiemannLiouville derivative, Applied Mathematics Letters 25 (2012) 1729-1733. doi:10.1016/j.aml.2012.02.001.

[5] Y. Khan, M. Fardi, K. Sayevand, M. Ghasemi, Solution of nonlinear fractional differential equations using an efficient approach, Neural Computing and Applications 24 (2014) 187-192. doi:10.1007/s00521-012-1208-7.

[6] A. K. Golmankhaneh, R. Arefi, D. Baleanu, Synchronization in a nonidentical fractional order of a proposed modified system, Journal of Vibration and Control 21 (2015) 1154-1161.

[7] Q. Li, T. Kang, Q. Zhang, Mean-square dissipative methods for stochastic age-dependent capital system with fractional Brownian motion and jumps, Applied Mathematics and Computation 339 (2018) 81-92. doi:10.1016/j.amc.2018.07.018.

[8] G.-C. Wu, D. Baleanu, W.-H. Luo, Lyapunov functions for RiemannLiouville-like fractional difference equations, Applied Mathematics and Computation 314 (2017) 228-236. doi:10.1016/j.amc.2017.06.019.

[9] D. Baleanu, G.-C. Wu, Y.-R. Bai, F.-L. Chen, Stability analysis of Caputolike discrete fractional systems, Communications in Nonlinear Science and Numerical Simulation 48 (2017) 520-530. doi:10.1016/j.cnsns.2017.01.002.

[10] T. T. Soong, Random Differential Equations in Science and Engineering, Academic Press, New York, 1973.

[11] T. Neckel, F. Rupp, Random Differential Equations in Scientific Computing, Versita, London, 2013.

[12] M. Khodabin, K. Maleknejad, M. Rostami, M. Nouri, Numerical solution of stochastic differential equations by second order Runge Kutta methods, Mathematical and Computer Modelling 59 (53) (2011) 1910-1920. doi:10.1016/j.mcm.2011.01.018.

[13] K. Nouri, H. Ranjbar, Mean square convergence of the numerical solution of random differential equations, Mediterranean Journal of Mathematics 12 (3) (2015) 1123-1140. doi:10.1007/s00009-014-0452-8.

[14] C. Wu, Y. Yang, Q. Zhao, Y. Tian, Z. Xua, Epidemic waves of a spatial SIR model in combination with random dispersal and non-local dispersal, Applied Mathematics and Computation 313 (2017) $122-143$. doi:10.1016/j.amc.2017.05.068.

[15] C. Burgos, J. C. Cortés, L. Villafuerte, R. J. Villanueva, Extending the deterministic Riemann-Liouville and Caputo operators to the random framework: A mean square approach with applications to solve random fractional differential equations, Chaos, Solitons \& Fractals 102 (2017) 305-318. doi:10.1016/j.chaos.2017.02.008.

[16] V. Lupulescu, D. O'Reagan, G. u. Rahman, Existence results for random fractional differential equations, Opuscula Mathematica 34 (4) (2014) 813-825. doi:10.7494/OpMath.2014.34.4.813.

[17] V. Lupulescu, K. N. Ntouyas, Random fractional differential equations, International Electronic Journal of Pure and Applied Mathematics 4 (2) (2012) 119-136

[18] M. Mazandarani, A. V. Kamyad, Modified fractional Euler method for solving fuzzy fractional initial value problem, Communications in Nonlinear Science and Numerical Simulation 18 (2013) 12-21. doi:10.1016/j.cnsns.2012.06.008.

[19] H. Vu, N. N. Phung, N. Phuong, On fractional random differential equations with delay, Opuscula Mathematica 36 (4) (2016) 541-556. doi:10.7494/OpMath.2016.36.4.541.

[20] S. Abbas, W. A. Albarakati, M. Benchohra, J. Henderson, Existence and Ulam stabilities for Hadamard fractional integral equations with random effects, Electronic Journal of Differential Equations 25 (2016) 1-12.

[21] S. Abbas, M. Benchohra, J.-E. Lazreg, G. M. N'Guérékata, Hilfer and Hadamard functional random fractional differential inclusions, CUBO: A Mathematical Journal 19 (1) (2017) 17-38.

[22] G. González-Parra, B. M. Chen-Charpentier, A. J. Arenas, Polynomial chaos for random fractional order differential equations, Applied Mathematics and Computation 226 (2014) 123-130. doi:10.1016/j.amc.2013.10.051.

[23] O. Vallée, M. Soares, Airy Functions and Applications to Physics, Imperial College Press, London, 2010. 
[24] A. Aghili, H. Zeinali, Solution to Fractional Schrodinger and Airy Differential Equations via Integral Transforms, British Journal of Mathematics \& Computer Sciences 4 (18) (2014) 2630-2664.

[25] V. Varlamov, Fractional derivatives of products of Airy functions, Journal of Mathematical Analysis and Applications 337 (1) (2008) 667-685.

[26] A. Ansari, The fractional Airy transform, Hacettepe Journal of Mathematics and Statistics 44 (4) (2015) 761-766

[27] J. C. Cortés, L. Jódar, J. Camacho, L. Villafuerte, Random Airy type differential equations: Mean square exact and numerical solutions, Computers and Mathematics with Applications 60 (2010) 1237-1244. doi:10.1016/j.camwa.2010.05.046

[28] J. C. Cortés, P. Sevilla-Peris, L. Jódar, Analytic-numerical approximating processes of diffusion equation with data uncertainty, Computers and Mathematics with Applications 49 (7-8) (2005) 1255-1266. doi:10.1016/j.camwa.2004.05.015.

[29] K. Diethelm, The analysis of fractional differential equations: An application-oriented exposition using differential operators of Caputo type, Springer, 2010.

[30] M. Loève, Probability Theory I, Vol. 45 of Graduate Texts in Mathematics, Springer-Verlag, New York, 1977.

[31] G. R. Grimmett, D. R. Stirzaker, Probability and Random Processes, Clarendon Press, New York, 2000.

[32] R. Pathria, P. Beale, Statistical Mechanics, Academic Press, 2011.

[33] J. Kapur, J. Kesavan, Entropy Optimization Principles with Applications, Academic Press, 1992.

[34] R. Gray, Entropy and Information Theory, Springer, 1990.

[35] A. Papoulis, S. U. Pillai, Probability, Random Variables and Stochastic Processes, 4th Edition, McGraw-Hill, New York, 2002.

[36] J. Borwein, Q. Zhu, Techniques of Variational Analysis, Springer-Verlag, 2005. 